\title{
ON SYMMETRIC QUADRANGULATIONS
}

\author{
MARIE ALBENQUE, ÉRIC FUSY AND DOMINIQUE POULALHON
}

\begin{abstract}
This note gathers observations on symmetric quadrangulations, with enumerative consequences. In the first part a new method for the enumeration of rooted simple quadrangulations is presented, based on quotienting symmetric simple quadrangulations in two different ways (one standard, the other one new). In the second part, based on results of Bouttier, Di Francesco and Guitter and on quotient and substitution operations, the series of several families of symmetric quadrangular dissections (general, without multiple edges, without nonfacial 4-cycle, respectively) is computed, with control on the distance from the central vertex to the outer boundary.
\end{abstract}

\section{INTRODUCTION}

A planar map is a connected graph embedded in the plane up to continuous deformation; the unique unbounded face of a planar map is called the outer face, the other ones are called inner faces. A map is said to be rooted if an edge of the outer face is marked and oriented so as to have the outer face on its left. A quadrangulation is a map with all faces of degree 4 . For $k \geq 1$, a quadrangular dissection of $a 2 k$-gon or $k$-dissection for short is a map whose outer face contour is a simple cycle of length $2 k$, and with all inner faces of degree 4. A map is said to be simple if it has no multiple edges; a $k$-dissection is called irreducible if every 4-cycle delimits a face (possibly the outer one).

The enumeration of families of rooted maps has received a lot of attention for more than 40 years; several methods can be applied: the recursive method introduced by Tutte [13], the random matrix method introduced by Brézin et al [3], and the bijective method introduced by Cori and Vauquelin [5] and Schaeffer [12]. In the first part of this note, we show another method for the enumeration of rooted simple quadrangulations based on quotienting symmetric simple quadrangulations. Historically, the enumeration of symmetric maps of order $k$ in a family $\mathcal{M}$ (i.e., such that a rotation of order $k$ fixes the map) was reduced to the enumeration of rooted maps in $\mathcal{M}$ via a quotient argument, a method used by Liskovets [9]. We proceed in the reverse way, namely we use symmetric simple quadrangulations to build an algebraico-differential equation satisfied by the series of rooted simple quadrangulations. Precisely the two sides of the equation correspond to two different ways of quotienting a symmetric map; one is classical, the other one is new and relies deeply on the existence and properties of $\alpha$-orientations which we recall in Section 1.1. From the functional equation we recover (by standard guessing/checking) that there are $\frac{4(3 n) !}{n !(2 n+2) !}$ rooted simple quadrangulations with $n+1$ faces.

Let us now describe the classical quotient operation, which is central in our work; for $k \geq 2$, a $k$-dissection $D$ is called $k$-symmetric if the plane embedding (conveniently deformed) is invariant by a rotation of angle $2 \pi / k$ centered at a vertex - called the center of the dissection. As observed by Liskovets [9], any two semi-infinite straight lines starting from the center and forming an angle of $2 \pi / k$ delimit a sector of $D$. When keeping only this sector and pasting these two lines together, we obtain a planar map, called the quotient-map of $D$; note that in our case, this map has outer degree 2.

The results in the second part of the note (rather independent from the first part) are expressions of the series of several families of symmetric quadrangular dissections with control on a distance parameter. Families of $k$-symmetric dissections have been counted according to the number of inner faces by Brown [4, 8] using the recursive method (Liskovet's quotient method [9] can also be applied, reducing the enumeration to rooted quadrangular dissections). In Section 2, combining results by Bouttier et al. [1] with the quotient method and substitution operations, we count - for any $k \geq 2-k$-symmetric general (resp. simple, irreducible) dissections according to the number of inner faces and the distance of the center from the outer boundary. This is the first result on a distance parameter for irreducible quadrangulations (which are well known to be in one-to-one correspondence with 3-connected maps); and it illustrates again the property that the series expression of a "well behaved" map family $\mathcal{M}$ refined by a distance parameter $d$ is typically expressed in terms of the $d$ th power of an algebraic series of

Adress: LIX, École Polytechnique, 91128 Palaiseau cedex, France; email: albenque,fusy,poulalhon@lix.polytechnique.fr. Supported by the European project ExploreMaps - ERC StG 208471. 


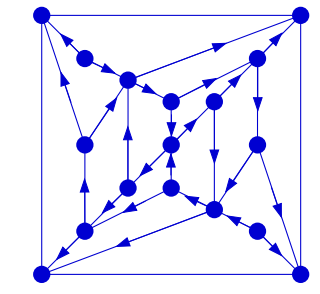

(a) Symmetric simple quadrangulation

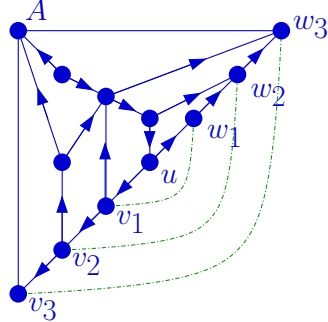

(b) Classical identification

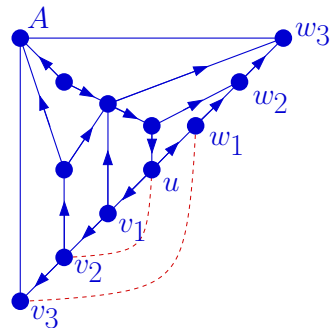

(c) New identification

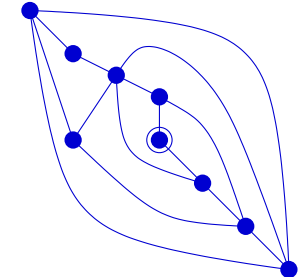

(d) Classical quotient

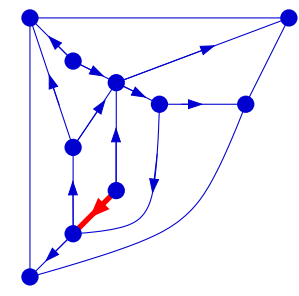

(e) New quotient

FIGURE 1. Starting from a symmetric simple quadrangulation endowed with its minimal 2-orientation (a), we can perform either a classical quotient (b), (d) or the new quotient (c), (e).

singularity type $z^{1 / 4}$ (implying that asymptotically the distance parameter $d$ on a random map of size $n$ in $\mathcal{M}$ converges in the scale $n^{1 / 4}$ as a random variable).

\section{ENUMERATION OF SIMPLE QUADRANGULATIONS VIA SYMMETRIC QUADRANGULATIONS}

In this section, simple quadrangulation are required to have at least 2 faces (to avoid the degenerated case with two edges and one face) and we call symmetric simple quadrangulations the simple 2-symmetric dissections. Edges and vertices of a map are called outer or inner depending on whether they are incident to the outer face or not.

1.1. A new way of quotienting a symmetric quadrangulation based on minimal 2-orientations. De Fraysseix and Ossona de Mendez [6] have shown that a quadrangulation is simple iff it admits an orientation of its inner edges so that inner vertices have outdegree 2 and outer ones have outdegree 0 ; such an orientation is called a 2-orientation. By a general result of Felsner [7] on orientations with prescribed outdegrees, any simple quadrangulation $Q$ admits a unique 2-orientation with no counterclockwise circuit, called the minimal 2-orientation of $Q$, see Figure 1(a). We now fix a 2-symmetric simple quadrangulation $Q$ and describe a new way of quotienting $Q$ relying on its minimal 2-orientation $O$. Note that unicity of the minimal 2-orientation implies that $O$ is itself stable by the $\pi$-rotation fixing $Q$. For each inner edge $e$ of $Q$, call leftmost path starting at $e$ the unique oriented path $P$ starting at $e$, ending at one outer vertex, and such that for any triple $v, v^{\prime}, v^{\prime \prime}$ of successive vertices along $P,\left(v^{\prime}, v^{\prime \prime}\right)$ is the first outgoing edge after $\left(v, v^{\prime}\right)$ in clockwise order around $v^{\prime}$. It can be shown (the proof, omitted here and working for any simple quadrangulation, is based on Euler relation, outdegrees and minimality) that the leftmost path is well defined and is a simple path ending at an outer vertex of $Q$.

Let $u$ be the central vertex, $e_{1}, e_{2}$ its two outgoing edges, and $P_{1}=\left(u=v_{0}, v_{1}, \ldots, v_{p}\right), P_{2}=(u=$ $\left.w_{0}, w_{1}, \ldots, w_{p}\right)$ the leftmost paths of $e_{1}$ and $e_{2}$ respectively. Since the minimal 2-orientation is stable by the $\pi$-rotation fixing $Q, e_{2}$ is clearly the image of $e_{1}$, and $P_{2}$ is the image of $P_{1}$ by this rotation. Hence $P_{1}$ and $P_{2}$ can not meet except at their starting point $u$. Call $P$ the union of $P_{1}$ and $P_{2}$; note that $P$ is a simple path connecting an outer vertex of $Q$ to a diagonally opposed outer vertex of $Q$, and $P$ is 


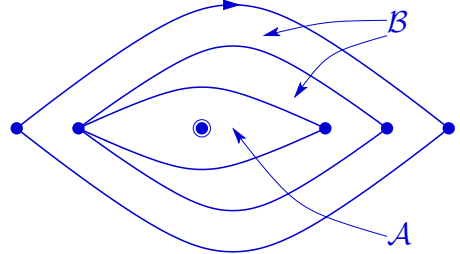

(a) Nested 2-cycles

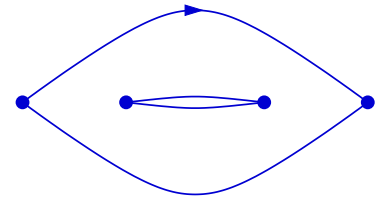

(b) Element of $\mathcal{B}$

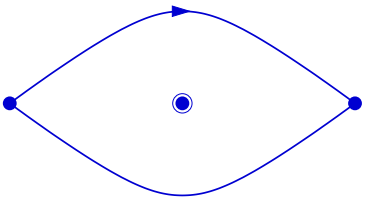

(c) Element of $\mathcal{A}$

FIGURE 2. The decomposition of a quasi-simple quadrangular 1-dissection

stable by the $\pi$-rotation fixing $Q$. Hence if we cut along $P$ we split $Q$ into two isomorphic dissections, see Figure 1(c). Let $Q_{1}$ be the one with clockwise contour $u, v_{1}, v_{2}, \ldots$ If $Q_{1}$ is a quadrangulation, we set $\Phi(Q):=Q_{1}$ and mark the edge $\left(u, v_{1}\right)$. Otherwise, for any $i \in\{0, \ldots, p-2\}$, we identify in $Q_{1}$ vertices $v_{i+2}$ with $w_{i}$, and merge corresponding edges; this defines the map $\Phi(Q)$, in which we then mark the edge $\left(v_{1}, v_{2}\right)$. Concerning orientations, the identification of $v_{i+2}$ with $w_{i}$ creates an orientation conflict only when merging $\left(u, v_{1}\right)$ with $\left(v_{1}, v_{2}\right)$. We choose to orient the merged edge from $v_{1}$ to $v_{2}$. With this convention, $\Phi(Q)$ is naturally endowed with a 2-orientation, and it can be checked that this 2-orientation is the minimal one and the leftmost path of the marked edge $\left(v_{1}, v_{2}\right)$ is $\left(v_{1}, v_{2}, \ldots, v_{p}\right)$. From these observations it is easy to describe the inverse mapping (mapping a simple quadrangulation with a marked edge to a symmetric simple quadrangulation). We obtain:

Theorem 1. The mapping $\Phi$ is a one-to-one correspondence between symmetric simple quadrangulations with $2 n$ inner faces and simple quadrangulations with $n$ inner faces and a marked edge.

1.2. Classical quotient vs. new quotient, and getting a functional equation. A quadrangular dissection is said to be pointed if it has a marked vertex. As any $k$-symmetric dissection is implicitly pointed (at the center), its $k$-quotient is a pointed 1-dissection (i.e. with outer face of degree 2). A pointed dissection is called quasi-simple if the marked vertex lies strictly in the interior of any 2-cycle. Then the following can be shown (see Lemma 5 in Section 2 for a more general statement):

Proposition 2. The 2-quotient is a one-to-one correspondence between symmetric simple quadrangulations with $2 n$ inner faces and quasi-simple pointed 1-dissections with $n$ inner faces.

Corollary 3. Simple quadrangulations with $n$ inner faces and a marked edge are in one-to-one correspondence with quasi-simple pointed 1-dissections with $n$ inner faces.

This correspondence allows us to get a functional equation for the generating series of rooted simple quadrangulations. Let us first observe that any edge of a simple quadrangulation has an implicit orientation given by the minimal 2-orientation of the quadrangulation. And a pointed 1-dissection also has an implicit rooting. Hence Corollary 3 is equivalent (by doubling each object) to: simple quadrangulations with $n$ inner faces and a marked oriented edge are in one-to-one correspondence with rooted quasi-simple pointed 1-dissections with $n$ inner faces.

Let us now define the family $\widetilde{\mathcal{Q}}$ of rooted simple quadrangulations, including the two degenerated ones with one face and two edges, and $\mathcal{Q}$ the family of nondegenerated ones; let $q(x)=\sum_{n \geq 2} q_{n} x^{n}$ and $\widetilde{q}(x)=\sum_{n \geq 1} q_{n} x^{n}$ be respectively the series of $\mathcal{Q}$ and of $\widetilde{\mathcal{Q}}$ according to the number of faces (including the outer one). Since $q_{1}=2, \widetilde{q}(x)=q(x)+2 x$. We will decompose both families involved in Corollary 3 in terms of $\mathcal{Q}$.

Let us first consider simple quadrangulations with a marked oriented edge. Any of them can equivalently be seen as a (nondegenerated) rooted simple quadrangulation with a marked face (possibly the outer one), hence their generating series according to inner faces is:

$$
\sum_{n \geq 2} n q_{n} x^{n-1}=q^{\prime}(x)
$$

On the other side, let $\mathcal{D}$ denote the family of rooted quasi-simple 1-dissections, and let us now describe how to decompose a dissection $D \in \mathcal{D}$ (an equivalent decomposition for symmetric 2-connected maps is described by Liskovets and Walsh [10]). First, observe that the separating 2-cycles of $D$ are nested, so we 
can consider these 2-cycles as forming an ordered sequence (from innermost to outermost). This yields a decomposition of $D$ as a sequence of components. Let $\mathcal{A}$ be the family of rooted pointed 1-dissections where the unique 2-cycle is the outer face, and let $\mathcal{B}$ be the family of rooted maps with all faces of degree 4 except for two special faces of degree 2 one of which is the outer face, and such that there is at least one quadrangular face and there is no other 2-cycle than the contours of the two special faces. We can cut $D$ along the nested 2-cycles to obtain a pointed 1-dissection in $\mathcal{A}$ and a sequence of maps in $\mathcal{B}$ (see Figure 2). Denoting respectively by $d(x), a(x), b(x)$ the series of $\mathcal{D}, \mathcal{A}$, and $\mathcal{B}$ according to the number of quadrangular faces, we obtain

$$
d(x)=\frac{a(x)}{1-b(x)}
$$

Starting from a dissection in $\mathcal{A}$, and deleting the unique non-root outer edge, one obtains a rooted quadrangulation in $\widetilde{\mathcal{Q}}$ with a pointed vertex not incident to the root-edge, and the mapping is invertible. Since a quadrangulation with $n$ faces has $n+2$ vertices (hence $n$ vertices not incident to the root-edge), we conclude that

$$
a(x)=\sum_{n \geq 1} n q_{n} x^{n}=2 x+x q^{\prime}(x) .
$$

Starting from a dissection in $\mathcal{B}$, contracting the innermost 2-cycle into a single marked edge, and deleting the unique non-root outer edge, one obtains a rooted quadrangulation in $\widetilde{\mathcal{Q}}$ with a marked non-root edge. Since a quadrangulation with $n$ faces has $2 n$ edges, we conclude that

$$
b(x)=\sum_{n \geq 1}(2 n-1) q_{n} x^{n}=2 x+2 x q^{\prime}(x)-q(x) .
$$

We finally obtain the equation

$$
q^{\prime}(x)=\frac{x \cdot\left(q^{\prime}(x)+2\right)}{1+q(x)-2 x-2 x q^{\prime}(x)},
$$

which can be rearranged into the following equation:

$$
x=\frac{q^{\prime}(x) \cdot(1+q(x))}{2+2 q^{\prime}(x)^{2}+3 q^{\prime}(x)} .
$$

From this equation, one readily extracts the development of $q(x)$ incrementally:

$$
q(x)=x^{2}+2 x^{3}+6 x^{4}+22 x^{5}+91 x^{6}+408 x^{7}+1938 x^{8}+\cdots .
$$

As shown next the exact expression of the coefficients (first obtained by Tutte from the recursive method [13] and subsequently recovered by Schaeffer [12] using a bijection with ternary trees) can also be recovered from (2):

Proposition 4. For $n \geq 1$ let $s_{n}$ be the number of rooted simple quadrangulations with $n$ faces. Then

$$
s_{n}=\frac{4(3 n) !}{n !(2 n+2) !} .
$$

Equivalently the series $q(x)=\sum_{n \geq 1} s_{n} x^{n+1}=\sum_{n \geq 2} q_{n} x^{n}$ is expressed as $q(x)=x \cdot\left(-\alpha^{2}+3 \alpha-2\right)$, where $\alpha \equiv \alpha(x)$ is specified by $\alpha=1+x \alpha^{3}$ ( $\alpha$ is the series of rooted ternary trees).

Proof. Equation (2) above admits a unique power series solution that is equal to 0 at 0 (indeed the equation is enough to extract the coefficients iteratively), so it remains to check that $f \equiv f(x):=$ $x \cdot\left(-\alpha^{2}+3 \alpha-2\right)$ is solution of $(2)$, i.e., to check that

$$
x=\frac{f^{\prime}(x) \cdot(1+f(x))}{2+2 f^{\prime}(x)^{2}+3 f^{\prime}(x)} .
$$

Note that $x, f(x)$ and $f^{\prime}(x)$ all have rational expressions in terms of $\alpha \equiv \alpha(x)$; after simple computations:

$$
x=\frac{\alpha-1}{\alpha^{3}}, \quad f(x)=\frac{\alpha-1}{\left(3 \alpha-\alpha^{2}+2\right) \alpha^{3}}, \quad f^{\prime}(x)=2 \alpha-2 .
$$

so it suffices to check that the expression of $x$ in terms of $\alpha$ (which is $(\alpha-1) / \alpha^{3}$ ) coincides with the expression $R(\alpha)$ of $f^{\prime}(x) \cdot(1+f(x)) /\left(2+2 f^{\prime}(x)^{2}+3 f^{\prime}(x)\right)$ in terms of $\alpha$. After simplification we find indeed that $R(\alpha)=(\alpha-1) / \alpha^{3}$, which concludes the proof. 


\section{Distance From the Central Vertex to the OUter Boundary}

For $k \geq 2, i>0$, and $\mathcal{D}^{(k)}$ a family of $k$-symmetric dissections, let $\mathcal{D}_{i}^{(k)}$ be the family of dissections in $\mathcal{D}^{(k)}$ where the centre is at distance $i$ from the outer face boundary (for instance, in the example of Figure 1(a), the central vertex is at distance 3 from the outer boundary). Let $a_{n, i}^{(k)}$ be the number of dissections in $\mathcal{D}_{i}^{(k)}$ with $n k$ inner faces; and let $D_{i}^{(k)}(x)=\sum_{n} a_{n, i}^{(k)}$ be the corresponding series. A dissection is called irreducible if all 4-cycles are the boundary of a face (possibly the outer one). We compute here in each $k \geq 2$ the expression of $D_{i}^{(k)}(x)$ for 3 classical families of quadrangulations: general, simple, and irreducible. We use the letter $\mathcal{F}, \mathcal{G}, \mathcal{H}$ (instead of $\mathcal{D})$ for the general, simple, and irreducible case respecively.

To obtain these expressions we combine results of Bouttier et al. [1,2] on the 2-point functions of quadrangulations with quotient and substitution operations. Our arguments rely on the following lemma whose proof is omitted here:

Lemma 5. For $k \geq 2$ let $D$ be a $k$-symmetric dissection, and $E$ the $k$-quotient of $D$ (both $D$ and $E$ are pointed dissections). Given a pointed dissection $P$ of pointed vertex $u$, denote by $o(P)$ the outer degree of $P, \ell(P)$ the length of a shortest cycle not equal to the outer face boundary and strictly enclosing $u$, $m(P)$ the length of a shortest cycle not strictly enclosing $u, \widetilde{m}(P)$ the length of a shortest non-empty cycle not strictly enclosing $u$, and $d(P)$ the distance of the pointed vertex to the outer boundary. Then

$$
o(D)=k \cdot o(E), \quad \ell(D)=k \cdot \ell(E), \quad m(D)=m(E), \quad \widetilde{m}(D)=\widetilde{m}(E), \quad d(D)=d(E) .
$$

Let $\mathbf{F}_{i}^{(k)}, \mathbf{G}_{i}^{(k)}, \mathbf{H}_{i}^{(k)}$ be respectively the images of $\mathcal{F}_{i}^{(k)}, \mathcal{G}_{i}^{(k)}, \mathcal{H}_{i}^{(k)}$ by the $k$-quotient operation. Note that the counting series $x \mapsto F_{i}^{(k)}(x)$ (resp. $\left.y \mapsto G_{i}^{(k)}(y), z \mapsto H_{i}^{(k)}(z)\right)$ of $\mathcal{F}_{i}^{(k)}\left(\operatorname{resp} . \mathcal{G}_{i}^{(k)}, \mathcal{H}_{i}^{(k)}\right.$ ) according to the number of orbites of inner faces is also the series of $\mathbf{F}_{i}^{(k)}$ (resp. $\mathbf{G}_{i}^{(k)}, \mathbf{H}_{i}^{(k)}$ ) according to the number of inner faces. By Lemma $5, \mathbf{F}_{i}^{(k)}$ is the family of pointed 1-dissections where the pointed vertex is at distance 1 from the outer face. Notice that the outer face can be contracted into a single edge, yielding a quadrangulation of the sphere with a marked edge $e$ and pointed vertex $v$ at distance $i$ from $e$. The counting series of such quadrangulations has been computed by Bouttier et al. [1], yielding the expression (not depending on $k$ ):

(3) $F_{i}^{(k)}(x)=X_{i}-X_{i-1}$, with $X_{i}=X_{\infty} \frac{\left(1-X^{i}\right)\left(1-X^{i+3}\right)}{\left(1-X^{i+1}\right)\left(1-X^{i+2}\right)}, \quad X+\frac{1}{X}+1=\frac{1}{x X_{\infty}^{2}}, X_{\infty}=1+3 x X_{\infty}^{2}$,

where $X, X_{i}, X_{\infty}$ are series in $x$, and the dependency between these series is $X_{\infty} \rightarrow X \rightarrow X_{i}$.

We now compute $y \mapsto G_{i}^{(k)}(y)$. By Lemma $5, \mathbf{G}_{i}^{(k)}$ is the family of quasi-simple pointed 1-dissections where the pointed vertex is at distance $i$ from the outer boundary (note that $\mathbf{G}_{i}^{(k)}$ does not depend on $k$ ). We proceed by substitution (an equivalent approach formulated on some labelled trees is discussed in [2]). The core of $\gamma \in \mathbf{F}_{i}^{(k)}$ is obtained by collapsing each 2-cycle of $\gamma$ not strictly enclosing the pointed vertex into a single edge; this yields a quasi-simple 1-dissection, and the distance of the pointed vertex $u$ to the outer boundary is still $i$ (because there is no way of shortening this distance by travelling inside a 2-cycle not enclosing $u$ ). Conversely each $\gamma \in \mathbf{F}_{i}^{(k)}$ is uniquely obtained from $\kappa \in \mathbf{G}_{i}^{(k)}-$ with $n$ inner faces - where each of the $2 n+1$ edges is either left unchanged or blown into a double edge in the interior of which a rooted quadrangulation is patched. Denoting by $f \equiv f(x)$ the series of rooted quadrangulations according to the number of faces, we obtain

$$
F_{i}^{(k)}(x)=\sum_{n \geq 1}\left[y^{n}\right] G_{i}^{(k)}(y) x^{n} \cdot(1+f)^{2 n+1}=(1+f) \cdot G_{i}^{(k)}\left(x \cdot(1+f)^{2}\right) .
$$

The series $f(x)$ is well known to be algebraic: $1+f=X_{\infty}-x X_{\infty}{ }^{3}$. Define $Y_{\infty}(y)$ as the series in $y$ such that $Y_{\infty}(y)=X_{\infty}(x) /(1+f)$ under the change of variable relation $y=x(1+f)^{2}$. Then it is easily checked that $Y_{\infty}(y)$ satisfies (and is specified by) the equation $Y_{\infty}=1+y Y_{\infty}{ }^{3}$ (see $[11,2]$ ). Define $Y(y)$ as $Y(y):=X(x)$ under $y=x(1+f)^{2}$. One easily checks that $x X_{\infty}(x)^{2}=y Y_{\infty}(y)^{2}$ when $y=x \cdot(1+f)^{2}$, hence $Y(y)$ satisfies (and is specified by) the equation $Y+1 / Y+1=1 /\left(y Y_{\infty}^{2}\right)$. Now define $Y_{i}(y)=X_{i}(x) /(1+f)$ under $y=x \cdot(1+f)^{2}$. Since $Y(y)=X(x)$ and $Y_{\infty}(y)=X_{\infty}(x) /(1+f)$, the expression of $X_{i}$ above ensures that

$$
Y_{i}=Y_{\infty} \frac{\left(1-Y^{i}\right)\left(1-Y^{i+3}\right)}{\left(1-Y^{i+1}\right)\left(1-Y^{i+2}\right)} .
$$


Since $G_{i}^{(k)}(y)=F_{i}^{(k)}(x) /(1+f)$ under the relation $y=x(1+f)^{2}$, and since $F_{i}^{(k)}(x)=X_{i}-X_{i-1}$, we have $G_{i}^{(k)}(y)=Y_{i}-Y_{i-1}$. To summarize we obtain the following expression for $G_{i}^{(k)}(y)$ (note that the expression does not depend on $k \geq 2$ ):

$$
G_{i}^{(k)}(y)=Y_{i}-Y_{i-1}, \text { with } Y_{i}=Y_{\infty} \frac{\left(1-Y^{i}\right)\left(1-Y^{i+3}\right)}{\left(1-Y^{i+1}\right)\left(1-Y^{i+2}\right)}, \quad Y+\frac{1}{Y}+1=\frac{1}{y Y_{\infty}^{2}}, Y_{\infty}=1+y Y_{\infty}^{3} .
$$

We now use a substitution approach at faces (instead of edges) to get an expression for $H_{i}^{(k)}(z)$ for $k \geq 2$ and $i>0$; this time the expressions for $k=2$ and for $k>2$ are different; we start with the case $k>2$ which is simpler. By Lemma $5, \mathbf{H}_{i}^{(k)}$ is the family of pointed 1-dissections where the 2-cycles and non-facial 4-cycles strictly enclose the pointed vertex $u$; we call such pointed dissections quasi-irreducible. The core of $\gamma \in \mathbf{G}_{i}^{(k)}$ is obtained by emptying each maximal (for the enclosed area) 4-cycle of $\gamma$ not strictly enclosing $u$; this yields a quasi-irreducible 1-dissection, and the distance of the pointed vertex $u$ to the outer boundary is still $i$ (because there is no way of shortening this distance by travelling inside a 4-cycle not enclosing $u$ ). Conversely each $\gamma \in \mathbf{G}_{i}^{(k)}$ is uniquely obtained from $\kappa \in \mathbf{H}_{i}^{(k)}$ where at each face a rooted (non-degenerated) simple quadrangulation is patched. Denoting by $g \equiv g(y)$ the series of rooted quadrangulations according to the number of inner faces, we obtain, for $k \geq 3$ and $i>0$ :

$$
G_{i}^{(k)}(y)=H_{i}^{(k)}(g(y)) .
$$

Again the series $g(y)$ is algebraic: $g(y)=y \cdot\left(-Y_{\infty}{ }^{2}+3 Y_{\infty}-2\right)$. Define $Z_{\infty} \equiv Z_{\infty}(z)$ as the series in $z$ specified by $Z_{\infty}(z):=Y_{\infty}(y)$ under the change of variable relation $z=g(y)$. It is easily checked (see [11]) that $Z_{\infty}(z)$ satisfies (and is specified by) the equation $Z_{\infty}(z)=1+z+\left(Z_{\infty}-1\right)^{2}$. Define $Z \equiv Z(z)$ as the series such that $Z(z):=Y(y)$ under the relation $z=g(y)$. Then it is easy to compute that $1 /\left(y Y_{\infty}^{2}\right)=1+1 /\left(Z_{\infty}-1\right)$ when $z=g(y)$, hence $Z$ is specified by $Z+1 / Z=1 /\left(Z_{\infty}-1\right)$. Now define $Z_{i} \equiv Z_{i}(z)$ as $Z_{i}(z):=Y_{i}(y)$ under $z=g(y)$. Since $Z(z)=Y(y)$ and $Z_{\infty}(z)=Y_{\infty}(y)$ under the relation $z=g(y)$, the expression of $Y_{i}$ above ensures that

$$
Z_{i}=Z_{\infty} \frac{\left(1-Z^{i}\right)\left(1-Z^{i+3}\right)}{\left(1-Z^{i+1}\right)\left(1-Z^{i+2}\right)} .
$$

Since $H_{i}^{(k)}(z)=G_{i}^{(k)}(y)$ under $z=g(y)$ and $G_{i}^{(k)}(y)=Y_{i}-Y_{i-1}$, we have $H_{i}^{(k)}(z)=Z_{i}-Z_{i-1}$. To summarize we obtain the following expression for $H_{i}^{(k)}(z)$ for $k \geq 3$ (again the expression does not depend on $k$ ):

$H_{i}^{(k)}(z)=Z_{i}-Z_{i-1}$, with $Z_{i}=Z_{\infty} \frac{\left(1-Z^{i}\right)\left(1-Z^{i+3}\right)}{\left(1-Z^{i+1}\right)\left(1-Z^{i+2}\right)}, \quad Z+\frac{1}{Z}=\frac{1}{Z_{\infty}-1}, Z_{\infty}=1+z+\left(Z_{\infty}-1\right)^{2}$.

For $k=2$ and $i>0$, by Lemma $5, \mathbf{H}_{i}^{(k)}$ is the family of pointed 1-dissections where the unique 2 -cycle is the outer boundary and where non-facial 4-cycles strictly enclose the pointed vertex $u$. These dissections are the cores of pointed 1-dissections where the unique 2-cycle is the outer boundary and the pointed vertex is at distance $i$ from the outer boundary. Contracting the outer 2-cycle, one gets a simple quadrangulation on the sphere with a marked edge $e$ and a pointed vertex at distance $i$ from $e$. The series $K_{i}(y)$ of such quadrangulations was computed by Bouttier and Guitter [2] $\left(\delta_{i, j}\right.$ is the Kronecker symbol):

$$
K_{i}(y)=\delta_{i, 1}-\left[u^{i-1}\right] \frac{1}{\sum_{i>0}\left(Y_{i+1} Y_{i}-Y_{i} Y_{i-1}\right) u^{i-1}} .
$$

The above substitution approach ensures that $K_{i}(y)=H_{i}^{(2)}(g(y))$. We obtain

$$
H_{i}^{(2)}(z)=\delta_{i, 1}-\left[u^{i-1}\right] \frac{1}{\sum_{i>0}\left(Z_{i+1} Z_{i}-Z_{i} Z_{i-1}\right) u^{i-1}} .
$$

\section{REFERENCES}

[1] J. Bouttier, P. Di Francesco, and E. Guitter. Geodesic distance in planar graphs. Nucl. Phys., B663:535-567, 2003.

[2] J. Bouttier and E. Guitter. Distance statistics in quadrangulations with no multiple edges and the geometry of minbus. J. Phys. A, A 43:313-341, 2010.

[3] E. Brézin, C. Itzykson, G. Parisi, and J.-B. Zuber. Planar diagrams. Comm. Math. Phys., 59:35-51, 1978.

[4] W.G. Brown. Enumeration of triangulations of the disk. Proc. London Math. Soc. (3), 14:746-768, 1964.

[5] R. Cori and B. Vauquelin. Planar maps are well labeled trees. Canad. J. Math., 33(5):1023-1042, 1981. 
[6] H. de Fraysseix and P. Ossona de Mendez. On topological aspects of orientations. Discrete Math., 229(1-3):57-72, 2001.

[7] S. Felsner. Lattice structures from planar graphs. Electron. J. Combin, Jan 2004.

[8] W.G. G Brown. Enumeration of quadrangular dissections of the disk. Canad. J. Math., 17:302-317, 1965.

[9] V. A. Liskovets. A census of non-isomorphic planar maps. In Colloq. Math. Soc. János Bolyai. 25th Algebraic Methods in Graph Theory, Vol. I, II, Szeged (Hungary), pages 479-484, 1978.

[10] V. A. Liskovets and T. R. S. Walsh. The enumeration of non-isomorphic 2-connected planar maps. Canad. J. Math., 35(3):417-435, 1983.

[11] R.C. Mullin and P.J. Schellenberg. The enumeration of c-nets via quadrangulations. J. Combin. Theory, 4:259-276, 1968.

[12] G. Schaeffer. Conjugaison d'arbres et cartes combinatoires aléatoires. PhD thesis, Université Bordeaux I, 1998.

[13] W. T. Tutte. A census of planar maps. Canad. J. Math., 15:249-271, 1963. 